$12-1-2021$

\title{
STROCSS 2021: Strengthening the reporting of cohort, cross- sectional and case-control studies in surgery
}

Ginimol Mathew

Riaz Agha

STROCSS Group

Joerg Albrecht

Prabudh Goel

See next page for additional authors

Follow this and additional works at: https://ecommons.aku.edu/pakistan_fhs_mc_surg_neurosurg

Part of the Neurosurgery Commons, Surgery Commons, and the Urology Commons 


\section{Authors}

Ginimol Mathew, Riaz Agha, STROCSS Group, Joerg Albrecht, Prabudh Goel, Indraneil Mukherjee, Prathamesh Pai, Anil K. D'Cruz, Ather Enam, and Hammad Ather 


\title{
STROCSS 2021: Strengthening the reporting of cohort, cross-sectional and case-control studies in surgery
}

\author{
Ginimol Mathew ${ }^{\mathrm{a}, *}$, Riaz Agha ${ }^{\mathrm{b}}$, STROCSS Group \\ ${ }^{\text {a }}$ York Teaching Hospital NHS Foundation Trust, York, United Kingdom \\ ${ }^{\mathrm{b}}$ Harley Clinic, London, United Kingdom
}

\section{A R T I C L E I N F O}

\section{Keywords:}

Cohort studies

Case-control studies

Cross-sectional studies

Reporting guideline

STROCSS

\begin{abstract}
A B S T R A C T
Introduction: Strengthening The Reporting Of Cohort Studies in Surgery (STROCSS) guidelines were developed in 2017 in order to improve the reporting quality of observational studies in surgery and updated in 2019. In order to maintain relevance and continue upholding good reporting quality among observational studies in surgery, we aimed to update STROCSS 2019 guidelines.

Methods: A STROCSS 2021 steering group was formed to come up with proposals to update STROCSS 2019 guidelines. An expert panel of researchers assessed these proposals and judged whether they should become part of STROCSS 2021 guidelines or not, through a Delphi consensus exercise.

Results: 42 people (89\%) completed the DELPHI survey and hence participated in the development of STROCSS 2021 guidelines. All items received a score between 7 and 9 by greater than $70 \%$ of the participants, indicating a high level of agreement among the DELPHI group members with the proposed changes to all the items.

Conclusion: We present updated STROCSS 2021 guidelines to ensure ongoing good reporting quality among observational studies in surgery.
\end{abstract}

\section{Introduction}

Observational studies often feature in the surgical literature [1]. However, poor reporting quality among observational studies in surgery has been highlighted [2]. In the absence of good reporting quality, readers are unable to meaningfully assess the research, rendering it less useful [3]. The existence of reporting guidelines and the mandatory implementation of these guidelines by journals have shown to improve the reporting quality among various types of studies [4-6].

Hence, Strengthening The Reporting Of Cohort Studies in Surgery (STROCSS) guidelines were developed in 2017 in order to improve the reporting quality of cohort studies in surgery. Despite the title, STROCSS guidelines aimed to improve the reporting quality of all observational studies in surgery, including case-control studies and cross-sectional studies, as well as cohort studies [7]. STROCSS 2017 guidelines were updated in 2019; since its inception, STROCSS guidelines have been cited over 1000 times illustrating their acceptance within the surgical research community [8]. We aimed to update STROCSS 2019 guidelines in order to maintain relevance and continue upholding good reporting quality among observational studies in surgery.

\section{Methods}

The DELPHI methodology used in the development of STROCSS 2017 and 2019 guidelines was used in the development of STROCSS 2021 guidelines [9].

\subsection{Coming up with proposals to update STROCSS 2019 guidelines}

A STROCSS 2021 steering group was formed; members collaborated over email, Google Docs and WhatsApp Messenger to come up with proposals to update STROCSS 2019 guidelines.

\subsection{Delphi process}

The proposals to update STROCSS 2019 guidelines were put to an expert panel of researchers; they were asked to assess the proposals and judge whether they should become part of STROCSS 2021 guidelines or not, through a Delphi consensus exercise.

The Delphi questionnaire was sent to all participants using Google Forms. The participants were required to indicate whether they disagreed or agreed with the proposed changes to the 17 items of the

\footnotetext{
* Corresponding author. York and Scarborough Teaching Hospitals NHS Foundation Trust, York Hospital, Wigginton Road, York, YO31 8HE, United Kingdom.

E-mail address: ginimol.mathew.13@ucl.ac.uk (G. Mathew).
} 
Table 1

STROCSS 2021 Delphi participants' scores ranging between 1 (strongly disagree) and 9 (strongly agree). Items listed correspond to individual sections of STROCSS.

\begin{tabular}{|c|c|c|c|}
\hline Item & $1-3(\%)$ & 4-6 (\%) & $7-9(\%)$ \\
\hline 1 & 2.4 & 7.2 & 90.5 \\
\hline $2 a$ & 0.0 & 2.4 & 97.6 \\
\hline $2 b$ & 0.0 & 9.6 & 90.4 \\
\hline $2 c$ & 2.4 & 7.2 & 90.5 \\
\hline $2 d$ & 0.0 & 19.1 & 81.0 \\
\hline 3 & 2.4 & 7.2 & 90.5 \\
\hline $4 a$ & 2.4 & 7.2 & 90.5 \\
\hline $4 \mathrm{~b}$ & 7.2 & 14.3 & 78.5 \\
\hline $4 c$ & 0.0 & 11.9 & 88.2 \\
\hline $4 d$ & 0.0 & 7.2 & 92.8 \\
\hline $5 a$ & 0.0 & 7.2 & 92.8 \\
\hline $5 b$ & 0.0 & 14.3 & 85.7 \\
\hline $5 c$ & 2.4 & 4.8 & 92.8 \\
\hline $5 d$ & 0.0 & 19.1 & 80.9 \\
\hline $6 a$ & 0.0 & 4.8 & 95.2 \\
\hline $6 b$ & 4.8 & 14.2 & 80.9 \\
\hline $6 c$ & 2.4 & 9.5 & 88.1 \\
\hline $7 a$ & 0.0 & 9.5 & 90.4 \\
\hline $7 \mathrm{~b}$ & 0.0 & 14.2 & 85.7 \\
\hline $7 c$ & 0.0 & 11.9 & 88.1 \\
\hline $7 d$ & 4.8 & 9.5 & 85.7 \\
\hline $7 e$ & 0.0 & 14.3 & 85.7 \\
\hline $7 f$ & 0.0 & 11.9 & 88.1 \\
\hline 8 & 0.0 & 9.5 & 90.5 \\
\hline 9 & 2.4 & 9.6 & 88.0 \\
\hline $10 \mathrm{a}$ & 0.0 & 2.4 & 97.6 \\
\hline $10 \mathrm{~b}$ & 0.0 & 9.5 & 90.4 \\
\hline $10 \mathrm{c}$ & 0.0 & 11.9 & 88.1 \\
\hline $11 \mathrm{a}$ & 0.0 & 19.0 & 80.9 \\
\hline $11 \mathrm{~b}$ & 0.0 & 16.7 & 83.4 \\
\hline $11 \mathrm{c}$ & 0.0 & 14.3 & 85.7 \\
\hline 12 & 0.0 & 9.6 & 90.4 \\
\hline 13 & 2.4 & 19.1 & 78.5 \\
\hline 14 & 0.0 & 9.5 & 90.5 \\
\hline 15 & 0.0 & 14.3 & 85.7 \\
\hline 16 & 2.4 & 14.3 & 83.3 \\
\hline $17 a$ & 2.4 & 14.3 & 83.3 \\
\hline $17 \mathrm{~b}$ & 0.0 & 4.8 & 95.2 \\
\hline $17 \mathrm{c}$ & 0.0 & 2.4 & 97.5 \\
\hline
\end{tabular}

STROCSS 2019 guidelines, using a nine-point Likert scale, where 1 indicated "strongly disagree" and 9 indicated "strongly agree". If greater than $70 \%$ of participants gave a score between 7 and 9 for a proposed change, this was deemed as consensus and the item was updated. If less than $70 \%$ of participants gave a score between 7 and 9 for a proposed change, the item was left unaltered.

\subsection{Participants}

Researchers who were involved in the development of STROCSS 2017 and 2019 guidelines were invited to participate again. In addition, members of the International Journal of Surgery (IJS) editorial board were invited; IJS has mandated authors submitting surgical research papers using observational methodology to comply with STROCSS guidelines and hence IJS is an ardent supporter of STROCSS guidelines. Participants were accomplished researchers, authors, journal reviewers, editorial board members and editors representing countries across North America, South America, Europe, Africa, Asia, and Australia.

\section{Results}

47 people agreed to participate in the development of STROCSS 2021 guidelines; 42 people (89\%) completed the DELPHI survey and hence participated in the development of STROCSS 2021 guidelines. Table 1 shows a summary of the scores given by the Delphi participants to indicate agreement or disagreement with the proposed changes to each item of the STROCSS 2019 guidelines. All items received a score
Table 2

The full revised STROCSS 2021 checklist.

\begin{tabular}{|c|c|c|}
\hline \multicolumn{3}{|c|}{ The STROCSS 2021 Guideline } \\
\hline $\begin{array}{l}\text { Item } \\
\text { no. }\end{array}$ & Item description & $\overline{\text { Page }}$ \\
\hline \multicolumn{3}{|c|}{ TITLE } \\
\hline 1 & $\begin{array}{l}\text { Title } \\
\text { - The word cohort or cross-sectional or case-control is included* } \\
\text { - Temporal design of study is stated (e.g. retrospective or } \\
\text { prospective) } \\
\text { - The focus of the research study is mentioned (e.g. population, } \\
\text { setting, disease, exposure/intervention, outcome etc.) } \\
\text { *STROCSS } 2021 \text { guidelines apply to cohort studies as well as } \\
\text { other observational studies (e.g. cross-sectional, case-control etc.) }\end{array}$ & \\
\hline \multicolumn{3}{|c|}{ ABSTRACT } \\
\hline $2 \mathbf{a}$ & $\begin{array}{l}\text { Introduction - briefly describe: } \\
\text { - Background } \\
\text { - Scientific rationale for this study } \\
\text { - Aims and objectives }\end{array}$ & \\
\hline 2b & $\begin{array}{l}\text { Methods - briefly describe: } \\
\text { - Type of study design (e.g. cohort, case-control, cross-sectional } \\
\text { etc.) } \\
\text { - Other key elements of study design (e.g. retro-/prospective, } \\
\text { single/multi-centred etc.) } \\
\text { - Patient populations and/or groups, including control group, if } \\
\text { applicable } \\
\text { - Exposure/interventions (e.g. type, operators, recipients, } \\
\text { timeframes etc.) } \\
\text { - Outcome measures - state primary and secondary outcome(s) }\end{array}$ & \\
\hline $2 c$ & $\begin{array}{l}\text { Results - briefly describe: } \\
\text { - Summary data with qualitative descriptions and statistical } \\
\text { relevance, where appropriate }\end{array}$ & \\
\hline 2d & $\begin{array}{l}\text { Conclusion - briefly describe: } \\
\text { - Key conclusions } \\
\text { - Implications for clinical practice } \\
\text { - Need for and direction of future research }\end{array}$ & \\
\hline \multicolumn{3}{|c|}{ INTRODUCTION } \\
\hline 3 & $\begin{array}{l}\text { Introduction - comprehensively describe: } \\
\text { - Relevant background and scientific rationale for study with } \\
\text { reference to key literature } \\
\text { - Research question and hypotheses, where appropriate } \\
\text { - Aims and objectives }\end{array}$ & \\
\hline \multicolumn{3}{|c|}{ METHODS } \\
\hline $4 a$ & Registration & \\
\hline
\end{tabular}

- In accordance with the Declaration of Helsinki*, state the research registration number and where it was registered, with a hyperlink to the registry entry (this can be obtained from ResearchRegistry.com, ClinicalTrials.gov, ISRCTN etc.)

- All retrospective studies should be registered before submission; it should be stated that the research was retrospectively registered

* "Every research study involving human subjects must be registered in a publicly accessible database before recruitment of the first subject" 4b Ethical approval

- Reason(s) why ethical approval was needed

- Name of body giving ethical approval and approval number

- Where ethical approval wasn't necessary, reason(s) are provided

4c Protocol

- Give details of protocol (a priori or otherwise) including how to access it (e.g. web address, protocol registration number etc.)

- If published in a journal, cite and provide full reference

Patient and public involvement in research

- Declare any patient and public involvement in research

- State the stages of the research process where patients and the public were involved (e.g. patient recruitment, defining

(continued on next page) 
Table 2 (continued)

\begin{tabular}{llc}
\hline \multicolumn{2}{l}{ The STROCSS 2021 Guideline } & Page \\
\hline $\begin{array}{l}\text { Item } \quad \text { Item description } \\
\text { no. }\end{array}$ & \\
\hline
\end{tabular}

research outcomes, dissemination of results etc.) and describe the extent to which they were involved.

5a Study design

- State type of study design used (e.g. cohort, cross-sectional, case-control etc.)

- Describe other key elements of study design (e.g. retro-/ prospective, single/multi-centred etc.)

5b Setting and timeframe of research - comprehensively describe:

- Geographical location

- Nature of institution (e.g. primary/secondary/tertiary care setting, district general hospital/teaching hospital, public/ private, low-resource setting etc.)

- Dates (e.g. recruitment, exposure, follow-up, data collection etc.)

5c Study groups

- Total number of participants

- Number of groups

- Detail exposure/intervention allocated to each group

- Number of participants in each group

5d Subgroup analysis - comprehensively describe:

- Planned subgroup analyses

- Methods used to examine subgroups and their interactions

Participants - comprehensively describe:

- Inclusion and exclusion criteria with clear definitions

- Sources of recruitment (e.g. physician referral, study website, social media, posters etc.)

- Length, frequency and methods of follow-up (e.g. mail, telephone etc.)

6b Recruitment - comprehensively describe:

- Methods of recruitment to each patient group (e.g. all at once, in batches, continuously till desired sample size is reached etc.)

- Any monetary incentivisation of patients for recruitment and retention should be declared; clarify the nature of any incentives provided

- Nature of informed consent (e.g. written, verbal etc.)

- Period of recruitment

6c Sample size - comprehensively describe:

- Analysis to determine optimal sample size for study accounting for population/effect size

- Power calculations, where appropriate

- Margin of error calculation

METHODS - INTERVENTION AND CONSIDERATIONS

7a Pre-intervention considerations - comprehensively describe:

- Preoperative patient optimisation (e.g. weight loss, smoking cessation, glycaemic control etc.)

- Pre-intervention treatment (e.g. medication review, bowel preparation, correcting hypothermia/-volemia/-tension, mitigating bleeding risk, ICU care etc.)

7b Intervention - comprehensively describe:

- Type of intervention and reasoning (e.g. pharmacological, surgical, physiotherapy, psychological etc.)

- Aim of intervention (preventative/therapeutic)

- Concurrent treatments (e.g. antibiotics, analgesia, antiemetics, VTE prophylaxis etc.)

- Manufacturer and model details, where applicable

7c Intra-intervention considerations - comprehensively describe:

- Details pertaining to administration of intervention (e.g. anaesthetic, positioning, location, preparation, equipment needed, devices, sutures, operative techniques, operative time etc.)

- Details of pharmacological therapies used, including

formulation, dosages, routes, and durations

- Figures and other media are used to illustrate
Table 2 (continued)

\begin{tabular}{|c|c|c|}
\hline \multicolumn{3}{|c|}{ The STROCSS 2021 Guideline } \\
\hline $\begin{array}{l}\text { Item } \\
\text { no. }\end{array}$ & Item description & Page \\
\hline $7 d$ & $\begin{array}{l}\text { Operator details - comprehensively describe: } \\
\text { - Requirement for additional training } \\
\text { - Learning curve for technique } \\
\text { - Relevant training, specialisation and operator's experience (e. } \\
\text { g. average number of the relevant procedures performed } \\
\text { annually) }\end{array}$ & \\
\hline $7 e$ & $\begin{array}{l}\text { Quality control - comprehensively describe: } \\
\text { - Measures taken to reduce inter-operator variability } \\
\text { - Measures taken to ensure consistency in other aspects of } \\
\text { intervention delivery } \\
\text { - Measures taken to ensure quality in intervention delivery }\end{array}$ & \\
\hline $7 f$ & $\begin{array}{l}\text { Post-intervention considerations - comprehensively describe: } \\
\text { - Post-operative instructions (e.g. avoid heavy lifting) and care } \\
\text { - Follow-up measures } \\
\text { - Future surveillance requirements (e.g. blood tests, imaging } \\
\text { etc.) }\end{array}$ & \\
\hline 8 & Outcomes - comprehensively describe: & \\
\hline
\end{tabular}

- Primary outcomes, including validation, where applicable

- Secondary outcomes, where appropriate

- Definition of outcomes

- If any validated outcome measurement tools are used, give full reference

- Follow-up period for outcome assessment, divided by group Statistics - comprehensively describe:

- Statistical tests and statistical package(s)/software used

- Confounders and their control, if known

- Analysis approach (e.g. intention to treat/per protocol)

- Any sub-group analyses

- Level of statistical significance

RESULTS

10a Participants - comprehensively describe:

- Flow of participants (recruitment, non-participation, crossover and withdrawal, with reasons). Use figure to illustrate.

- Population demographics (e.g. age, gender, relevant socioeconomic features, prognostic features etc.)

- Any significant numerical differences should be highlighted Participant comparison

- Include table comparing baseline characteristics of cohort groups

- Give differences, with statistical relevance

- Describe any group matching, with methods

$10 \mathrm{c}$

Intervention - comprehensively describe:

- Degree of novelty of intervention

- Learning required for interventions

- Any changes to interventions, with rationale and diagram, if appropriate

11a Outcomes - comprehensively describe:

- Clinician-assessed and patient-reported outcomes for each group

- Relevant photographs and imaging are desirable

- Any confounding factors and state which ones are adjusted

Tolerance - comprehensively describe:

- Assessment of tolerability of exposure/intervention

- Cross-over with explanation

- Loss to follow-up (fraction and percentage), with reasons

11c

Complications - comprehensively describe:

- Adverse events and classify according to Clavien-Dindo classification*

- Timing of adverse events

- Mitigation for adverse events (e.g. blood transfusion, wound care, revision surgery etc.)

(continued on next page) 
Table 2 (continued)

\begin{tabular}{|c|c|c|}
\hline \multicolumn{3}{|c|}{ The STROCSS 2021 Guideline } \\
\hline $\begin{array}{l}\text { Item } \\
\text { no. }\end{array}$ & Item description & Page \\
\hline 12 & $\begin{array}{l}\text { *Dindo D, Demartines N, Clavien P-A. Classification of Surgical } \\
\text { Complications. A New Proposal with Evaluation in a Cohort of } \\
6336 \text { Patients and Results of a Survey. Ann Surg. 2004; 240(2): } \\
205-213 \\
\text { Key results - comprehensively describe: } \\
\text { - Key results with relevant raw data } \\
\text { - Statistical analyses with significance } \\
\text { - Include table showing research findings and statistical analyses } \\
\text { with significance }\end{array}$ & \\
\hline \multicolumn{3}{|c|}{ DISCUSSION } \\
\hline 13 & $\begin{array}{l}\text { Discussion - comprehensively describe: } \\
\text { - Conclusions and rationale } \\
\text { - Reference to relevant literature } \\
\text { - Implications for clinical practice } \\
\text { - Comparison to current gold standard of care } \\
\text { - Relevant hypothesis generation }\end{array}$ & \\
\hline 14 & $\begin{array}{l}\text { Strengths and limitations - comprehensively describe: } \\
\text { - Strengths of the study } \\
\text { - Weaknesses and limitations of the study and potential impact } \\
\text { on results and their interpretation } \\
\text { - Assessment and management of bias } \\
\text { - Deviations from protocol, with reasons }\end{array}$ & \\
\hline 15 & $\begin{array}{l}\text { Relevance and implications - comprehensively describe: } \\
\text { - Relevance of findings and potential implications for clinical } \\
\text { practice } \\
\text { - Need for and direction of future research, with optimal study } \\
\text { designs mentioned }\end{array}$ & \\
\hline \multicolumn{3}{|c|}{ CONCLUSION } \\
\hline 16 & $\begin{array}{l}\text { Conclusions } \\
\text { - Summarise key conclusions } \\
\text { - Outline key directions for future research }\end{array}$ & \\
\hline DECL & TIONS & \\
\hline $17 \mathbf{a}$ & Conflicts of interest & \\
\hline $17 b$ & $\begin{array}{l}\text { - Conflicts of interest, if any, are described } \\
\text { Funding }\end{array}$ & \\
\hline $17 \mathrm{c}$ & $\begin{array}{l}\text { - Sources of funding (e.g. grant details), if any, are clearly stated } \\
\text { - Role of funder } \\
\text { Contributorship } \\
\text { - Acknowledge patient and public involvement in research; } \\
\text { report the extent of involvement of each contributor }\end{array}$ & \\
\hline
\end{tabular}

between 7 and 9 by greater than $70 \%$ of the participants, indicating consensus with the proposed changes to all the items. The revised STROCSS 2021 guidelines are shown in Table 2.

\section{Discussion}

Since the publication of STROCSS guidelines, it has been cited over 1000 times and thus enjoyed great acceptance within the surgical research community. We present the updated STROCSS 2021 guidelines to continue ensuring good reporting quality among observational studies in surgery; we encourage authors, reviewers, editors, and journals to adopt them.

Authors should cite STROCSS 2021 guidelines in their methods section; additionally, they should submit a completed STROCSS 2021 guidelines checklist alongside their manuscript for reviewers and editors to inspect and ensure compliance. STROCSS website (https://www.st rocssguideline.com) has provided the STROCSS 2021 guidelines checklist in various formats to ensure accessibility.

\section{Conclusion}

We present updated STROCSS 2021 guidelines for authors, reviewers, editors, and journals to implement, with a view to ensuring good reporting quality among observational studies in surgery.

\section{Funding}

None.

\section{Ethical approval}

Not applicable.

\section{Sources of funding}

None.

\section{Author contribution}

RA: Concept and design, data interpretation and analysis, drafting, revision and approval of final manuscript. GM: Design, data collection, data interpretation and analysis, drafting, revision and approval of final manuscript.

\section{Research registration Unique Identifying number (UIN)}

1. Name of the registry: Not applicable.

2. Unique Identifying number or registration ID: Not applicable.

3. Hyperlink to your specific registration (must be publicly accessible and will be checked): Not applicable.

\section{Guarantor}

Riaz Agha.

\section{Data statement}

The data in this guideline is derived from individual responses to the DELPHI survey, and so is confidential and not in the public domain.

\section{Declaration of competing interest}

None declared - the authors have no financial, consultative, institutional, and other relationships that might lead to bias or conflict of interest.

\section{Acknowledgements}

Michelle Griffin, Stanford University, Palo Alto, United States.

\section{References}

[1] J. Song, K. Chung, Observational studies: cohort and case-control studies, Plast. Reconstr. Surg. 126 (6) (2010) 2234-2242.

[2] R. Agha, S. Lee, K. Jeong, A. Fowler, D. Orgill, Reporting quality of observational studies in plastic surgery needs improvement, Ann. Plast. Surg. 76 (5) (2016) $585-589$.

[3] E. von Elm, D. Altman, M. Egger, S. Pocock, P. Gøtzsche, J. Vandenbroucke, Strengthening the reporting of observational studies in epidemiology (STROBE) statement: guidelines for reporting observational studies, PLoS Med. 4 (10) (2007) 296.

[4] R. Agha, R. Farwana, M. Borrelli, T. Tickunas, T. Kusu-Orkar, M. Millip, R. Thavayogan, J. Garner, D. Orgill, Impact of the SCARE guideline on the reporting of surgical case reports: a before and after study, Int. J. Surg. 45 (2017) 144-148.

[5] R. Agha, M. Borrelli, R. Farwana, T. Kusu-Orkar, M. Millip, R. Thavayogan, J. Garner, N. Darhouse, D. Orgill, Impact of the PROCESS guideline on the reporting of surgical case series: a before and after study, Int. J. Surg. 45 (2017) 92-97.

[6] R. Agha, A. Fowler, C. Limb, K. Whitehurst, R. Coe, H. Sagoo, D. Jafree, C. Chandrakumar, B. Gundogan, Impact of the mandatory implementation of 
reporting guidelines on reporting quality in a surgical journal: a before and after study, Int. J. Surg. 30 (2016) 169-172.

[7] R. Agha, M. Borrelli, M. Vella-Baldacchino, R. Thavayogan, D. Orgill, STRocss Group, The STROCSS statement: strengthening the reporting of cohort studies in surgery, Int. J. Surg. 46 (2017) 198-202.
[8] R. Agha, A. Abdall-Razak, E. Crossley, N. Dowlut, C. Iosifidis, G. Mathew, STROCSS Group, STROCSS 2019 guideline: strengthening the reporting of cohort studies in surgery, Int. J. Surg. 72 (2019) 156-165.

[9] J. Pill, The Delphi method: substance, context, a critique and an annotated bibliography, Soc. Econ. Plann. Sci. 5 (1) (1971) 57-71. 